\title{
Direct Observation and Analysis of Spin Dependent Transport of Single Atoms in a 1D Optical Lattice
}

\author{
Michał Karski, Leonid Förster, Andrea Alberti, Wolfgang Alt, Artur Widera and Dieter Meschede* \\ Institut für Angewandte Physik, Universität Bonn \\ Jai-Min CHOI \\ Department of Science Education, Chonbuk National University, Jeonju 561-756, Korea
}

\begin{abstract}
We have directly observed spin-dependent transport of single cesium atoms in a 1D optical lattice. A superposition of two circularly polarized standing waves is generated from two counter propagating, linearly polarized laser beams. Rotation of one of the polarizations by $\pi$ causes displacement of the $\sigma^{+}$and the $\sigma^{-}$-lattices by one lattice site. Unidirectional transport over several lattice sites is achieved by rotating the polarization back and forth and flipping the spin after each transport step. We have analyzed the transport efficiency over 10 and more lattice sites, and we discuss and quantify the relevant error sources.
\end{abstract}

PACS numbers: 05.60.Gg, 37.10.Jk, 37.10.Vz

Keywords: Optical lattices, Cold atoms, Quantum transport

\section{INTRODUCTION}

Controlled transport of atoms stored in optical lattices is a central process in the quest for coherent atom-atom interactions. Such interactions are at the heart of creating and manipulating quantum multi-particle systems for, e.g., quantum simulations. Spin-dependent transport - where the direction of transport depends on the spin state - opens especially interesting routes because it allows the creation of spin-position entangled quantum states as precursors for correlated many-particle states. A concept for such a transport with neutral atoms stored in an optical lattice was proposed by Deutsch and Jessen and by Jaksch et al. [1,2] and first demonstrated by Mandel et al. [3] with a sample of ultracold rubidium atoms in a Mott insulator state, where the signature of transport was observed in momentum space.

Here, we report a direct observation of spin-dependent transport in a 1D optical lattice through fluorescence imaging. In contrast to the "top-down" approach of Ref. 3 we use single cesium atoms prepared in low-energy thermal states in deep lattice sites [4].

\section{SPIN-DEPENDENT TRANSPORT}

In a spin-dependent transport, the shift direction of a trapped atom along the lattice axis is determined by its

*E-mail: meschede@uni-bonn.de; Fax: +49-228-733474 internal spin (or qubit) state $|s\rangle=|\uparrow\rangle$ or $|\downarrow\rangle$. Such transport can be realized using two counterpropagating linearly polarized laser beams in a lin- $\theta$-lin configuration [2], in which the rotation angle $\theta$ of the polarization vector of one of the laser beams is continuously varied (see Fig. 1). The resulting light field can be decomposed into a $\sigma^{+}$and a $\sigma^{-}$circularly polarized standing wave, contributing to the trapping potential by $U_{+}(z, \theta)=U_{0} \cos (k z-\theta / 2)$ and $U_{-}(z, \theta)=U_{0} \cos (k z+\theta / 2)$, respectively. Here, $k=2 \pi / \lambda$ is the wave vector component along the lattice axis, and $U_{0}$ is the depth of the potential wells. By varying the rotation angle $\theta$, both standing waves and their contributions to the trapping potential are spatially shifted in opposite directions by a distance of $z_{ \pm}= \pm \theta / \pi \cdot \lambda / 4$, resulting in a relative displacement of $\Delta z=z_{+}-z_{-}=\theta / \pi \cdot \lambda / 2$ and an overlapping at $\theta / \pi=0, \pm 1, \pm 2, \ldots$. For the outermost Zeeman sublevels of the cesium hyperfine ground state manifold, a magic wavelength $\lambda_{\mathrm{m}}$ in between the $\mathrm{D}_{1}$ and the $\mathrm{D}_{2}$ lines can be found such that one spin state only experiences the $U_{+}(z, \theta)$ component of the trapping potential while the other spin state is mainly affected by the $U_{-}(z, \theta)$ component.

In our experiment, the rotation angle $\theta$ is voltagecontrolled using an electro-optical modulator (EOM) (see Fig. 1). The EOM is driven by a high-voltage amplifier with a $-3 \mathrm{~dB}$ bandwidth of $370 \mathrm{kHz}$ and a maximum output voltage of $750 \mathrm{~V}$, limiting the rotation angle range to $0 \leq \theta \leq 1.7 \pi$. For the spin (or qubit) states, we use $|\uparrow\rangle=\left|F=4, m_{\mathrm{F}}=4\right\rangle$ and $|\downarrow\rangle=\left|F=3, m_{\mathrm{F}}=3\right\rangle$. For the optical lattice, we choose $\lambda \equiv \lambda_{\mathrm{m}}=865.9 \mathrm{~nm}$ and $U_{0} / k_{\mathrm{B}}=80 \mu \mathrm{K}$, resulting in 


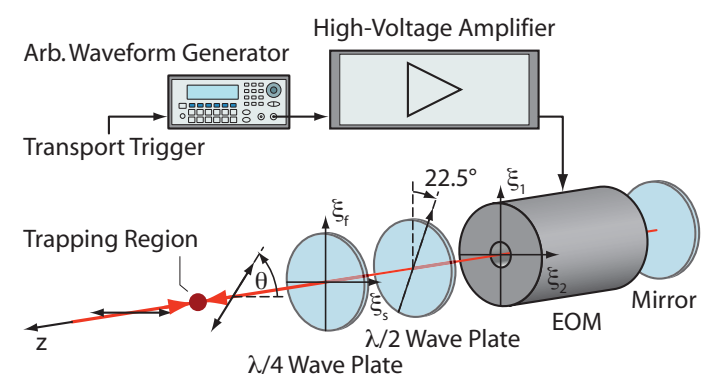

Fig. 1. (Color online) Experimental setup to control the rotation angle $\theta$ between the polarization vectors of an incident and a retro-reflected linearly polarized beam using an electro-optical modulator (EOM). Both principle axes of the quarter-wave plate, $\xi_{\mathrm{f}}$ and $\xi_{\mathrm{s}}$ (fast and slow axes), and those of the EOM, $\xi_{1}$ and $\xi_{2}$, are parallel and perpendicular to the polarization vector of the incident beam, respectively. A halfwave plate in between is used to rotate the incoming polarization to $45^{\circ}$ with respect to the principal axes of the EOM.

spin-dependent trapping potentials $U_{\uparrow}(z, \theta)=U_{+}(z, \theta)$ and $U_{\downarrow}(z, \theta)=\frac{1}{8} U_{+}(z, \theta)+\frac{7}{8} U_{-}(z, \theta)$ with axial and radial trapping frequencies of $\omega_{\mathrm{ax}}=2 \pi \times 115 \mathrm{kHz}$ and $\omega_{\text {rad }}=2 \pi \times 1 \mathrm{kHz}$, respectively. Due to the residual contribution of the $U_{+}(z, \theta)$ component to the $U_{\downarrow}(z, \theta)$ potential, its shape changes in depth and contrast during the shift, affecting the axial and the radial trapping frequencies of atoms transported in state $|\downarrow\rangle$. Furthermore, the spatial shift of $U_{\downarrow}(z, \theta)$ depends nonlinearly on the rotation angle $\theta$, see, e.g., Ref. 1 .

The limited range of the rotation angle allows only two perfectly overlapping trapping potential configurations: namely, $\theta=0$ and $\theta=\pi$, where $U_{\uparrow}(z, \theta)=U_{\downarrow}(z, \theta)$. We, therefore, define a single transport step as a shift by a distance of $\pm \lambda / 4$, i.e., from one overlapping configuration to another. Taking into account the periodicity of the optical lattice, it is convenient to discretize the position space along the lattice axis in units of $\lambda / 4$ (see Fig. 2). The spin-dependent shift operators then read

$$
\hat{S}_{\mathrm{fw}}:\left\{\begin{array}{l}
|\uparrow, l\rangle \rightarrow e^{i \varphi_{\uparrow}}|\uparrow, l+1\rangle \\
|\downarrow, l\rangle \rightarrow e^{i \varphi_{\downarrow}}|\downarrow, l-1\rangle
\end{array}\right.
$$

and

$$
\hat{S}_{\mathrm{bw}}:\left\{\begin{aligned}
|\uparrow, l\rangle & \rightarrow e^{i \varphi_{\uparrow}}|\uparrow, l-1\rangle \\
|\downarrow, l\rangle & \rightarrow e^{i \varphi_{\downarrow}}|\downarrow, l+1\rangle,
\end{aligned}\right.
$$

where we use a short-hand notation of spin-position product states $|s, l\rangle \equiv|s\rangle \otimes|l\rangle$ with $s=\{\uparrow, \downarrow\}$ and $l=0, \pm 1, \pm 2, \ldots$. The subscript of $\hat{S}$ indicates the rotation direction of the polarization, i.e., forward ( $\mathrm{fw}$ ) for $0 \rightarrow \pi$ and backward (bw) for $\pi \rightarrow 0$, shifting the spindependent potentials from one overlapping configuration to another. A spin-dependent phase $\varphi_{s}$ is accumulated during the shift with $\varphi_{\uparrow} \neq \varphi_{\downarrow}$.

We implement the shift operators $\hat{S}_{\mathrm{fw}}$ and $\hat{S}_{\mathrm{bw}}$ by feeding cosinusoidal driving ramps $V_{\mathrm{fw}}(t) \equiv V_{0}+\left(V_{\pi}-V_{0}\right)[1-$

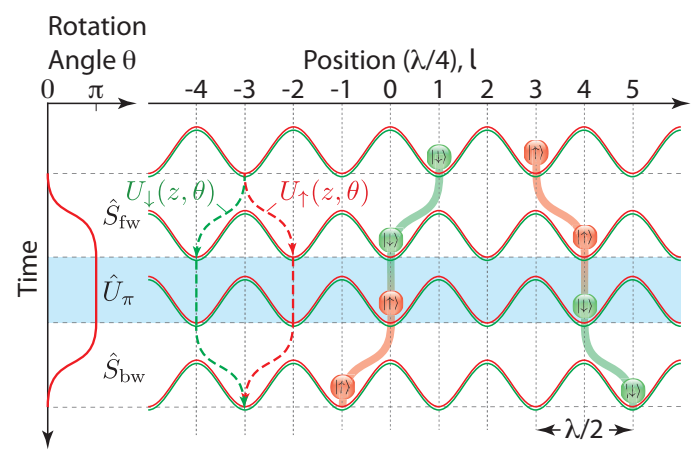

Fig. 2. (Color online) Schematic representation of unidirectional spin-dependent transport of atoms over distances of one lattice site. Shifts of the spin-dependent potentials $U_{\uparrow}(z, \theta)$ and $U_{\downarrow}(z, \theta)$ are indicated by dashed lines; and transport of atoms by shaded bold lines.

$\cos (\pi \cdot t / \tau)]$ and $V_{\mathrm{bw}}(t) \equiv V_{\mathrm{fw}}(\tau-t)$ to the amplifier's input, where $\tau$ denotes the ramp time, and $V_{0}$ and $V_{\pi}$ are the input voltages corresponding to the $\theta=0$ and $\theta=\pi$ configuration, respectively. For ramp times $\tau>14 \mu \mathrm{s}$, these ramps almost perfectly translate to the rotation angle $\theta(t)$. The ramp time is chosen such that excitations between vibrational states of the atoms are negligible while still being sufficiently fast to finish the experimental sequence within the phase coherence time of $T_{2} \approx 1 \mathrm{~ms}$ - a mandatory requirement for advanced applications of spin-dependent transport [5,6]. We calculate the excitation probability by using first-order perturbation theory [7], where the trapping potentials are approximated as harmonic, and the axial and radial dynamics of the atoms are assumed to be decoupled. For the cosinusoidal driving ramps, we find an optimum ramp time of $\tau=30 \mu \mathrm{s}$, resulting in an excitation probability of less than $3 \%$ per shift.

Spin-dependent transport over distances of $L=$ $1,2,3, \ldots$ lattice sites to the left or to the right (i.e., by $\pm L \cdot \lambda / 2)$ is realized by alternating the applications of the shift operators $\hat{S}_{\mathrm{fw}}$ and $\hat{S}_{\mathrm{bw}}$ with $\pi$-pulses flipping the spin states between $\left(\hat{U}_{\pi}:|s, l\rangle \rightarrow i|-s, l\rangle\right)$, see Fig. 2:

$$
\begin{aligned}
& \hat{T}_{2 L} \equiv\left(\hat{U}_{\pi} \hat{S}_{\mathrm{bw}} \hat{U}_{\pi} \hat{S}_{\mathrm{fw}}\right)^{L} \\
& \hat{T}_{2 L}:\left\{\begin{array}{l}
|\uparrow, l\rangle \rightarrow(-1)^{L} e^{i\left(\varphi_{\uparrow}+\varphi_{\downarrow}\right) L}|\uparrow, l+2 L\rangle \\
|\downarrow, l\rangle \rightarrow(-1)^{L} e^{i\left(\varphi_{\uparrow}+\varphi_{\downarrow}\right) L}|\downarrow, l-2 L\rangle .
\end{array}\right.
\end{aligned}
$$

The operator $\hat{T}_{2 L}$ is intentionally constructed from an even number of transport steps. By this, the entire transport sequence always starts and ends in the $\theta=0$ configuration, which, due to technical issues, is the experimentally most robust configuration providing long-term stability on the time scale of seconds. Such long-term stability is required for detection of the initial and the final positions of the atoms in the lattice by using fluorescence imaging [8] with a typical exposure time of $1 \mathrm{~s}$. Otherwise, because the trap depth for atoms, except those in 
$\left|F=4, m_{\mathrm{F}}=4\right\rangle$, is reduced in the non-overlapping case, atom losses would be enhanced during irradiation with the near-resonant light required for the imaging process.

The $\pi$-pulses are driven by resonant rectangular microwave pulses at $2 \pi \times 9.2 \mathrm{GHz}$ with a pulse duration of $8 \mu \mathrm{s}$ (Rabi frequency of $\approx 2 \pi \times 60 \mathrm{kHz}$ ). Alternatively, broadband composite pulses, so called $90_{0} 225_{180} 315_{0^{-}}$ pulses $[9,10]$ with a duration of $24 \mu$ s are employed to compensate for possible errors in the pulse frequency or power. To minimize frequency broadening from vectorial and tensorial contributions to the differential light shift, microwave transitions between the internalstates of the atoms are only induced in perfectly overlapping trapping potentials. The microwave pulses are applied after each shift operation with a time delay of $2 \mu \mathrm{s}$, which allows lattice polarization transients to settle. The latter are caused by the limited bandwidth of the polarization control setup and by excitation of mechanical resonances of the EOM crystal. The time delay has been determined using microwave spectroscopy, utilizing the fact that spectra are significantly broadened by any displacement of the $U_{\uparrow}, U_{\downarrow}$ potentials. This method ensures a maximum deviation of $|\Delta \theta|<10^{-2}$ for the rotation angle in the overlap configuration, corresponding to a maximum relative displacement of the spin-dependent trapping potentials of less than $1 \mathrm{~nm}$ from perfect overlap.

\section{MEASUREMENTS AND DISCUSSION}

To investigate the efficiency of our spin-dependent transport over distances of several lattice sites, we load an average of eight atoms into the optical lattice, which are randomly distributed over a region of about 150 lattice sites. We determine the initial positions of the atoms by fluorescence imaging (exposure time of $1 \mathrm{~s}$ ) and prepare them in the spin state $|\uparrow\rangle$ by using optical pumping. Details on state-preparation and detection can be found in Refs. 8 and 11. We subsequently apply the transport sequence defined by $\hat{T}_{2 L}$ for $L=\{1,2, \ldots, 11\}$, each composed of an even number of $2 L$ transport steps (see Eq. (3)). After transporting the atoms, we determine their final positions by fluorescence imaging and calculate their transport distances, i.e. the final positions relative to the initial ones, by considering only those atoms that are initially sufficiently far separated so that even in case of transport errors, their transport paths cannot cross. In Fig. 3, probability histograms of transport distances for different numbers of transport steps $2 L$ are shown. Each histogram has been normalized to the total number of analyzed atoms $(500-1000)$. Digitized histograms with a bin width of $\lambda / 2$ reveal the probability $P_{2 j}$ of finding an atom transported over a distance of $j \cdot \lambda / 2(j=0, \pm 1, \pm 2, \ldots)$. Densely sampled histograms with a bin width of $\lambda / 20$ reveal Gaussian peaks centered at integer multiples of $\lambda / 2$. These peaks clearly reproduce the periodicity of the optical lattice verifying the

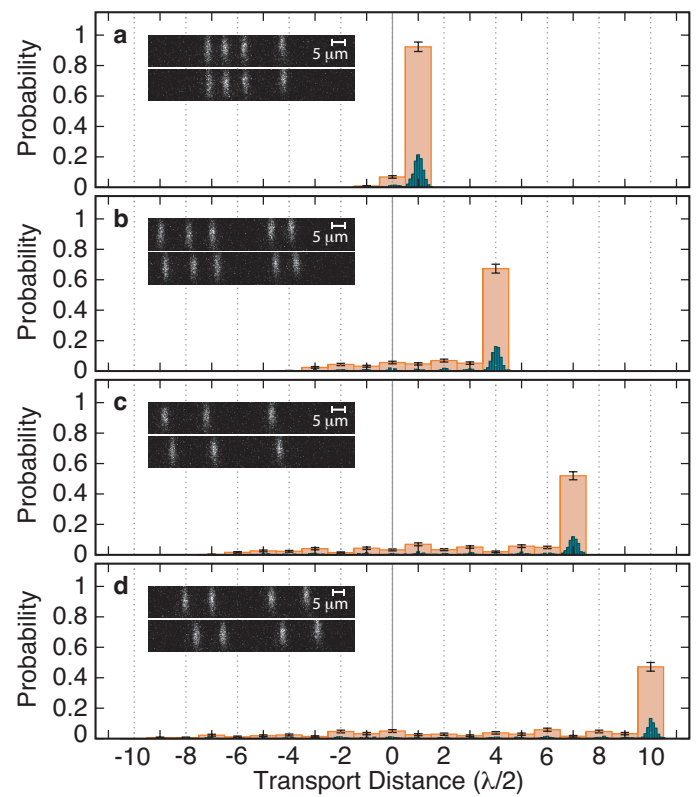

Fig. 3. (Color online) Probability histograms of transport distances of atoms initialized in the spin state $|\uparrow\rangle$ after a spin-dependent transport comprised $2 L$ transport steps for (a) $L=1$, (b) $L=4$, (c) $L=7$ and (d) $L=10$. Digitized histograms (light shade) with a bin width of $\lambda / 2$ indicate the transport distances in units of lattice sites. Densely sampled histograms (dark shade) with a bin width of $\lambda / 20$ reveal the periodicity of the optical lattice, indicating the absence of significant lattice drifts during the transport sequence. Insets show initial (upper) and final (lower) fluorescence sample images of transported atoms.

position resolution achieved in our fluorescence detection and indicating that no significant drifts of the optical lattice, relative to the imaging optics occurred during the transport sequence. From the Gaussian peaks, we estimate the reliability of inferring the correct transport distance $j \cdot \lambda / 2$, yielding $>99.7 \%$.

In the ideal case, starting from state $|\uparrow\rangle$ and applying $2 L$ transport steps, we expect only a single histogram bar at transport distance $L \cdot \lambda / 2$ with probability $P_{2 L}=100 \%$. We, indeed, observe the majority of atoms at the expected transport distance, however, with a reduced probability. The transport efficiency for $2 L$ transport steps is the measured probability for an atom to arrive at the nominal transport distance. Note that this definition does not contain any statement regarding the coherence properties of the transport. It solely reveals the successful displacement of atoms by $L$ lattice sites.

At a temperature of $10 \mu \mathrm{K}$ and a potential depth of $k_{\mathrm{B}} \times 80 \mu \mathrm{K}$, tunneling of atoms is extremely improbable during shifts of the spin-dependent potentials $\left(\approx 10^{-4}\right.$ per shift, inferred from band-structure calculation). For our analysis, we, therefore, assume perfect shift operations $\hat{S}_{\mathrm{fw}}$ and $\hat{S}_{\mathrm{bw}}$ and attribute the imperfections to the preparation and the evolution of internal states of atoms. 


\section{State Initialization and Photon Scattering}

Errors in the state initialization at the beginning or scattering of photons from the light field of the optical lattice during the transport sequence may transfer the atoms into spin states outside the Hilbert space of the qubit, i.e., to $\left|F=4, m_{\mathrm{F}} \neq 4\right\rangle$ or $\left|F=3, m_{\mathrm{F}} \neq 3\right\rangle$. Except for $m_{\mathrm{F}}=0$, atoms in these states effectively still experience a spin-dependent trapping potential due to an unequal fraction of potential contributions $U_{+}(z, \theta)$ and $U_{-}(z, \theta)$, however, with a reduced trap depth at $\theta=\pi / 2$. Hence, for atoms in states $\left|m_{\mathrm{F}}\right|<3$, we expect significant atom losses during the transport sequence. More importantly, once the internal states of the atoms have left the Hilbert space of the qubit, their possible transition frequencies are far detuned from the preset $\pi$-pulse frequency by at least $2 \pi \times 1 \mathrm{MHz}$. These atoms are then no longer affected by the microwave pulses of the transport sequence. For an even number of transport steps, they are henceforth transported back and forth close to the lattice site at which their internal state left the Hilbert space of the qubit for the first time, staying behind the nominal transport distance and thus reducing the transport efficiency. In contrast to the scattering of photons, which can be regarded as a per-step imperfection, state initialization is a one time operation that is performed only once at the beginning of each transport sequence. The corresponding error imposes, therefore, merely an upper limit on the transport efficiency, irrespective of the number of subsequent transport steps, $P_{2 L} \leq P_{\text {ini }}$.

In our experiment, state initialization is limited by the optical pumping efficiency, yielding $P_{\text {ini }}>97 \%$. The scattering of photons from the light field of the optical lattice with a calculated Raman and Rayleigh scattering rates of $10 \mathrm{~s}^{-1}$ and $5 \mathrm{~s}^{-1}$, respectively, is negligible at the time scale of a typical transport step with a probability of the order of $10^{-4}$. The effect of photon scattering on the transport efficiency is, thus, neglected.

\section{Perturbations of $\pi$-pulses}

Typical pulse errors can be divided into static and dynamic perturbations of pulse area, frequency and phase during the pulse. In ensemble averages, these perturbations usually contribute to inhomogeneous (static perturbations) or homogeneous (dynamic perturbations) broadenings or shifts. Their effect on the transport efficiency $P_{2 L}$ can be calculated by replacing the ideal $\pi$ pulse operators $\hat{U}_{\pi}$ in Eq. (3) by their perturbed counterparts.

Static phase perturbations do not affect the transport efficiency, because spin states of correctly transported atoms are automatically prepared in the pure basis states $|\uparrow\rangle$ or $|\downarrow\rangle$ by the shift operations. The same holds for phases accumulated during the shift so that the transport efficiency is insensitive to shift-induced dephasing.

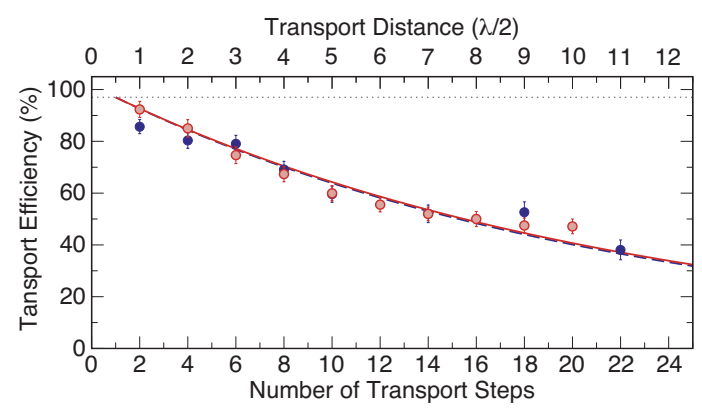

Fig. 4. (Color online) Transport efficiency $P_{2 L}$ as a function of the number of transport steps $2 L$ for a sequence employing rectangular $180_{0}(\pi)$-pulses $(\circ)$ and composite $90_{0} 225_{180} 315_{0}$-pulses of equal Rabi frequency $(\bullet)$. The vertical, dotted line indicates the upper transport efficiency limit imposed by the state initialization $P_{\text {ini. }}$. The solid (dashed) line shows a fit of the model function of Eq. (4) to the data obtained for $180_{0}$-pulses $\left(90_{0} 225_{180} 315_{0}\right.$-pulses) in the transport sequence.

Static perturbations of pulse area and frequency, on the other hand, directly affect the spin-flip efficiency in each step and, thus, the transport efficiency $P_{2 L}$ as well. Such perturbations, for instance, are induced by the radial oscillation of atoms in the trapping potential, which in our case may be regarded as frozen on the time scale of a single pulse [12]. Similarly, the thermal distribution over the axial vibrational states causes an inhomogeneous frequency broadening and induces a static perturbation of the Rabi frequency and, thus, of the pulse area via the Franck-Condon factor [13]. Vibrational excitation during the shift operations may even increase these inhomogeneities. From the transport efficiency alone, however, static perturbations are not only indistinguishable from one another but also from different dynamical perturbations such as repeatable drifts or fluctuations of lattice depth and polarization or magnetic fields. We, therefore, subsume all these perturbations in an effective spin-flip efficiency $\bar{P}_{\text {flip }}$ per pulse.

Assuming that $\bar{P}_{\text {flip }}$ does not significantly change over the entire transport sequence, we expect the transport efficiency to exponentially decay according to

$$
P_{2 L}^{\text {err }}=P_{\text {ini }} \cdot\left(\bar{P}_{\text {flip }}\right)^{2 L-1} .
$$

The alignment procedure of the experimental setup aims at identical microwave spectra for both overlapping configurations, $\theta=0$ and $\theta=\pi$, including the amplitudes, positions and shapes of the resonance peaks, supporting this assumption.

In Fig. 4, the measured transport efficiency as a function of the number of transport steps is shown. It fits well the exponential dependence of Eq. (4), yielding

$$
\bar{P}_{\text {flip }}=(95.5 \pm 0.3) \% .
$$

Slight deviations of individual data points from the fit in Fig. 4 could be partially attributed to slow drifts of lat- 
tice polarization over time. Such drifts have been monitored independently by measuring the polarization state of the retro-reflected beam after passing the trapping region of the atoms.

To reduce possible effects of static frequency and pulse area perturbations on the spin-flip efficiency, including the corresponding inhomogeneous broadening mechanisms, we have replaced the regular, rectangular $\pi$-pulses by broadband composite pulses in the transport sequence, namely $90_{0} 225_{180} 315_{0}$-pulses. The latter are robust against static detunings within a calculated ( $P_{\text {flip }} \geq 95 \%$-)range of $|\delta|<2 \pi \times 54 \mathrm{kHz}$ (compared to $|\delta|<2 \pi \times 14 \mathrm{kHz}$ of the regular, rectangular $\pi$-pulses) for static pulse area perturbations of up to $\pm 10 \%$. The measured transport efficiency as a function of the number of transport steps for this case is shown in Fig. $4(\bullet)$. From a fit of Eq. (4), we obtain

$$
\bar{P}_{\text {flip }, \mathrm{CP}}=(95.5 \pm 0.3) \%,
$$

which incidentally agrees with the value for the regular $\pi$-pulses (see Eq. (5)). This means that the compensation of static perturbations (e.g., inhomogeneities) by the composite pulses does not improve the transport efficiency. This finding suggests that the finite transport efficiency is dominated by dynamic perturbations, such as lattice polarization drifts and fluctuations (decoherence) during the pulse affecting the internal state of the atom. Also, we cannot exclude that the improvement of the spin-flip efficiency of the composite pulse is counteracted by additional dephasing during the pulse due to its three times longer duration.

\section{Dynamic Perturbations and Decoherence}

Decoherence is typically characterized by a longitudinal relaxation time $T_{1}$ and a dephasing time $T_{2}$. We have measured longitudinal relaxation by preparing the atoms in state $|\uparrow\rangle$ or $|\downarrow\rangle$ and performing a typical transport whereby $\pi$-pulses are removed from the sequence, and thus their possible errors as well. Atoms are then only transported back and forth to the initial lattice site. Finally, we determine the population in state $|\downarrow\rangle$ after different times by using state-selective push out [11], yielding $T_{1} \approx 100 \mathrm{~ms}$, in agreement with the calculated limit imposed by the Raman scattering processes in the lattice. Measuring dephasing during the pulses in the transport sequence and precisely inferring the irreversible $T_{2}^{\prime}$ time, e.g., from decay of Rabi oscillations, turns out to be non-trivial due to technical instability of the $\theta=\pi$ overlapping configuration on the time scale required. We, therefore, estimate this $T_{2}^{\prime}$ time by solving the (pulsedriven) optical Bloch equations for the measured spinflip efficiency of Eqs. (5) and (6), yielding $T_{2}^{\prime} \approx 100 \mu \mathrm{s}$. The corresponding spectrum, calculated from the optical Bloch equations, deviates, however, from the measured spectra in shape. These deviations may arise from

\begin{tabular}{lr}
\hline \hline Error source & Probability \\
\hline A. One time errors & \\
\hline Transport distance detection & $<0.3 \%$ \\
State initialization & $<3 \%$ \\
\hline B. Per step errors & $0.01 \%$ \\
\hline Tunneling of atoms & $0.01 \%$ \\
Raman scattering & \\
Imperfection of $\pi$ pulses & $<4.5 \%$ \\
- Regular rectangular pulses & $<4.5 \%$ \\
- Broadband composite pulses & $<1.1 \%$ \\
- For comparison: no transport & $<3 \%$ \\
Vibrational excitations & \\
\hline \hline
\end{tabular}

Table 1. Compilation of error probabilities (shift + pulse) limiting the measured transport efficiency or affecting the quantum state fidelity.

repeated (non-random) dynamic perturbations that are not covered by the simple relaxation model in the Bloch equations. Consequently, these perturbations should not be interpreted as fluctuations, and the true irreversible dephasing time $T_{2}^{\prime}$ may be much longer then estimated above. In comparison, in a "static" lattice configuration (no transport), this dephasing time $T_{2}^{\prime}$ is on the order of several milliseconds, as inferred from Rabi oscillations. We conclude from this fact that the spin-flip efficiency in the transport sequence is, indeed, reduced by dynamic perturbations, probably caused by drifts or oscillations of the lattice polarization, which possibly have not yet fully decayed after each shift, when the pulses are applied. On the one hand, such dynamic perturbation directly translate into drifts and fluctuations of differential light shifts, leading to homogeneous broadening of the transition frequency and, thus, dephasing. On the other hand, they translate into drifts and fluctuations of the Rabi frequency and, thus, of the pulse area via the Franck-Condon factor [13]. Our interpretation is, furthermore, supported by the higher spin-flip efficiency of $(98.9 \pm 0.2) \%$ measured in a "static" optical lattice. Employing optimum control techniques [14], either by microwave pulse-shaping or active polarization control, might help to counteract or compensate for dynamical drifts of our system, and, thus, improve the pulse efficiency in the future.

\section{SUMMARY}

We have directly observed and analyzed the transport efficiency of a unidirectional spin-dependent transport of single cesium atoms in a 1D optical lattice. Relevant error sources are compiled in Table 1. For our experimental parameters, tunneling of atoms is negligible during the transport, and excitations between vibrational states are minimized by numerically finding an optimum 
ramp time for a chosen ramp. Photon scattering processes and errors in state initialization play marginal roles while successful displacement of the atoms is insensitive to dephasing during the shift. The transport efficiency is mainly limited by the evolution of the internal states of the atoms. Our analysis of static and dynamic pulse perturbations suggests that spin-flip efficiencies are limited by repeated drifts or fluctuations during the transport operations.

\section{ACKNOWLEDGMENTS}

The authors gratefully acknowledge financial support by the Deutscher Akademischer Austauschdienst exchange program (KOSEF), the Deutsche Forschungsgemeinschaft FOR635, Me971/25-2, and the European Commission (IP AQUTE). M. Karski acknowledges support from the Studienstiftung des deutschen Volkes, J. M. Choi received partial support from a Korea Research Foundation grant funded by the Korean Government (Ministry of Education, MOEHRD).

\section{REFERENCES}

[1] I. H. Deutsch and P. S. Jessen, Phys. Rev. A 57, 1972 (1998).
[2] D. Jaksch, H. J. Briegel, J. I. Cirac, C. W. Gardiner and P. Zoller, Phys. Rev. Lett. 82, 1975 (1999).

[3] O. Mandel, M. Greiner, A. Widera, T. Rom, T. W. Hänsch and I. Bloch, Nature 425, 937 (2003).

[4] W. Alt, D. Schrader, S. Kuhr, M. Müller, V. Gomer and D. Meschede, Phys. Rev. A 67, 033403 (2003).

[5] O. Mandel, M. Greiner, A. Widera, T. Rom, T. W. Hänsch and I. Bloch, Phys. Rev. Lett. 91, 010407 (2003).

[6] M. Karski, L. Förster, J. M. Choi, A. Steffen, W. Alt, D. Meschede and A. Widera, Science 325, 174 (2009).

[7] W. Hänsel, J. Reichel, P. Hommelhoff and T. W. Hänsch, Phys. Rev. A 64, 063607 (2001).

[8] M. Karski, L. Förster, J. M. Choi, W. Alt, A. Widera and D. Meschede, Phys. Rev. Lett. 102, 053001 (2009).

[9] Z. Starčuk and V. Sklenář, J. Magn. Reson. 62, 113 (1985).

[10] M. H. Levitt, Composite Pulses, in Encyclopedia of $\mathrm{Nu}$ clear Magnetic Resonance (Wiley, New York, 1996).

[11] M. Karski, L. Förster, J. M. Choi, A. Steffen, N. Belmechri, W. Alt, D. Meschede and A. Widera, New J. Phys. 12, 065027 (2010).

[12] S. Kuhr, W. Alt, D. Schrader, I. Dotsenko, Y. Miroshnychenko, A. Rauschenbeutel and D. Meschede, Phys. Rev. A 72, 023406 (2005).

[13] L. Förster, M. Karski, J. M. Choi, A. Steffen, W. Alt, D. Meschede, A. Widera, E. Montano, J. H. Lee, W. Rakreungdet and P. S. Jessen, Phys. Rev. Lett. 103, 233001 (2009).

[14] G. De Chiara, T. Calarco, M. Anderlini, S. Montangero, P. J. Lee, B. L. Brown, W. D. Phillips and J. V. Porto, Phys. Rev. A 77, 052333 (2008). 